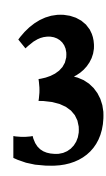

\title{
LA REPETICIÓN DE CURSO A DEBATE: UN ESTUDIO EMPÍRICO A PARTIR DE PISA 2015
}

(DEBATE ON GRADE REPETITION:

AN EMPIRICAL STUDY FROM PISA 2015)

Delia Arroyo Resino

Universidad Internacional de La Rioja

Israel Alexander Constante Amores

Inmaculada Asensio Muñoz

Universidad Complutense de Madrid

DOI: $10.5944 / e d u c X X 1.22479$

Cómo referenciar este artículo/How to reference this article:

Arroyo Resino, D.; Constante Amores, I. A. y Asensio Muñoz, I. (2019). La repetición de curso a debate: un estudio empírico a partir de PISA 2015. Educación XX1, 22(2), 69-92, doi: 10.5944/ educXX1.22479

Arroyo Resino, D.; Constante Amores, I. A. \& Asensio Muñoz, I. (2019). Debate on grade repetition: an empirical study from PISA 2015. Educación XX1, 22(2), 69-92, doi: 10.5944/ educXX1.22479

\section{RESUMEN}

La repetición de algún curso académico es una de las grandes preocupaciones del sistema educativo español, porque su tasa duplica el promedio de los países de la OCDE y la Unión Europea, lo que resulta muy costoso. Además, es una medida de calidad controvertida, ya que, lejos de lograr la nivelación pretendida, aparece asociada en la investigación con el fracaso escolar. En este trabajo se pretende estudiar empíricamente el fenómeno, identificando las variables que definen el perfil del estudiante repetidor y no repetidor y aquellos predictores que más afectan a la probabilidad de repetir. Para ello, se ha partido del cuestionario de contexto del estudiante proporcionado por el informe PISA 2015 de la OCDE para España, que ofrece información sobre variables vinculadas 
con los antecedentes del estudiante, procesos y medidas no cognitivas, y que permite identificar claramente al alumnado que ha repetido algún curso en primaria y/o secundaria y al que no. Los datos se han analizado con la técnica de árboles de decisión y mediante el modelo de regresión logística binaria. Los resultados muestran que las variables que mejor discriminan entre los perfiles del estudiante repetidor y del no repetidor son las aspiraciones educativas y el haber cursado una materia de ciencias el curso anterior. La principal conclusión del estudio es que la probabilidad de repetir se asocia más con variables de procesos (tiempo de aprendizaje y currículum) que con variables vinculadas a los antecedentes del estudiante, en contra de lo que se ha venido perpetuando a través de la literatura. Esto supone un distanciamiento respecto a los predictores que tradicionalmente se han relacionado con la repetición y la aportación de evidencia empírica favorable a la apertura de una nueva perspectiva educativa, basada en el abordaje de la repetición de curso desde un enfoque menos socioeconómico y más pedagógico.

\section{PALABRAS CLAVE}

PISA; repetición; minería de datos; regresión logística; variables de contexto.

\section{ABSTRACT}

Grade repetition is one of the major concerns in the Spanish educational system because its rate doubles the average performance of both OECD member countries and European countries. Grade repetition is usually linked to school failure and dropout instead of bringing student's academic level onto an equal plane. This research empirically identifies the variables which repeating and non-repeating students have in common and those possible variables which have a higher influence in retaking a year. Thus, the student context questionnaire development provided by OECD PISA 2015 for Spain has been used. These questionnaires provide information on variables linked to the student's background and also processes and non-cognitive outcomes. All of this information allows for the identification of the characteristics of both repeating and non-repeating students. Decision tree techniques and binary logistic regression have been applied to analyze the data. Results show that the educational aspirations and the fact of having studied a scientific module during the previous academic year are the main variables. The main finding of this study is that the probability of repeating an academic year is more influenced by process variables (learning time and curriculum) than by the student's background. 
This outcome is contrary to what has been perpetuated through literature and starts a new educational perspective regarding grade repetition from a pedagogical point of view and a lower socioeconomic focus.

\section{KEYWORDS}

PISA; repetition; data mining; regression logistics; context variables.

\section{INTRODUCCIÓN}

Según resultados obtenidos en el Programa para la Evaluación Internacional de Alumnos (PISA), uno de los principales problemas del sistema educativo español es la alta tasa de repetición (Méndez \& Cerezo, 2018), pues el 31\% de los estudiantes de 15 años han repetido en educación obligatoria (OCDE, 2016a). Dicho porcentaje es alarmante, ya que representa más del doble del promedio de la Unión Europea (15\%) y de la OCDE (12\%). Estas cifras sugieren que uno de los objetivos primordiales en el ámbito de la educación sea llegar a reducir dicha tasa (OCDE, 2016a), porque la repetición es una medida costosa, de efectividad dudosa y con efectos secundarios, lo que se traduce en que pocas veces favorece que los estudiantes afectados maduren y lleguen a conseguir los objetivos mínimos fijados para un curso o etapa y, sin embargo, se asocia de manera sistemática con niveles de desempeño menor.

La importancia de la repetición como tópico de investigación no radica únicamente en su alta incidencia, sino en que se relaciona con el bajo rendimiento, con el abandono educativo temprano y, en consecuencia, con el fracaso escolar (Benito, 2007; Choi, Gil, Mediavilla \& Valbuena, 2018), que representa otro de los grandes hándicaps del sistema educativo español (Crespo, 2018), llevando a la Comisión Europea (2010) a fijarse como objetivo su reducción al 10\% para el año 2020. La consecución de esta meta requiere de estudios rigurosos que permitan conocer a fondo variables que afectan al fracaso escolar, entre las cuales se encuentra el exceso de estudiantes repetidores, con el propósito de reducir su incidencia (Cordero, Manchón \& Simancas, 2014). En el presente trabajo, a partir de los datos de España en PISA 2015, se pretende dar respuesta a los siguientes problemas de investigación: ¿cuál es el perfil del estudiante repetidor y no repetidor que emerge de la minería en las bases de datos de PISA? y ¿qué variables de contexto (antecedentes del estudiante, procesos y medidas no cognitivas) se asocian con una mayor probabilidad de repetir en los estudiantes? 


\section{Repetición, fracaso escolar y variables de contexto}

La repetición de curso viene siendo objeto de discusión prácticamente desde el momento en que se instauraron las escuelas graduadas (Carabaña, 2013). En general, se ha venido utilizando como una estrategia de nivelación en los estudiantes que no alcanzan los objetivos mínimos (Cordero et al., 2014), aunque con resultados no siempre satisfactorios. Normalmente, la decisión sobre la repetición depende de los centros educativos, pero tiene un componente político y cultural importante, por lo que difiere de unos países a otros. Así, por ejemplo, en Japón y Noruega se ha prohibido la repetición y en Finlandia y Reino Unido solo se repite en circunstancias muy excepcionales.

En España, con la Ley Orgánica de Calidad de la Educación (LOCE, 2002) se planteó la eliminación de la promoción automática establecida por la Ley de Ordenación General del Sistema Educativo (LOGSE, 1990). Con la actual Ley Orgánica para la Mejora de la Calidad de la Educación (LOMCE, 2013) se establece que en la etapa de educación primaria los estudiantes podrán repetir como máximo un curso (artículo 20.2) y en secundaria (artículo 28) repetirán curso los estudiantes que hayan suspendido tres o más materias o dos asignaturas troncales, a no ser que el profesorado determine lo contrario. En esta última etapa educativa, el alumnado puede repetir el mismo curso una sola vez y dentro de la misma etapa como máximo dos veces, a excepción de cuarto de ESO que se puede repetir dos veces. Además, en dicha ley se establece el Programa de Mejora del Aprendizaje y el Rendimiento (PMAR), la Formación Profesional Básica (FPB) y el refuerzo de los conocimientos instrumentales de los estudiantes, con el fin de limitar la repetición de curso y el riesgo de fracaso escolar.

En los cambios habidos en el marco legal que la sustenta se refleja la falta de convicción en su efectividad. Por otra parte, los resultados de la investigación educativa de los últimos años sobre el tema asocian la repetición de curso con el fracaso escolar, al provocar efectos secundarios negativos, ya que suelen conducir a los estudiantes afectados hacia a una baja autoestima, y conlleva con cierta frecuencia problemas de absentismo, así como un abandono escolar temprano (Choi, 2017; Jacob \& Lefgren, 2009; Manacorda, 2012). Asensio, Carpintero, López y Expósito (2018), con datos de PISA 2015 para España, encuentran que efectivamente, la repetición se asocia con el bajo rendimiento, y que los estudiantes repetidores obtienen peores puntuaciones en ciencias y en matemáticas, siendo el haber repetido en secundaria la tercera variable de contexto más importante en la explicación de diferencias en el nivel de desempeño, después del grado internacional en que el estudiante está escolarizado (variable que también implícitamente informa de la repetición) y de las expectativas del alumno. La evidencia a nivel 
internacional va en esta línea. Un meta-análisis realizado por Jimerson (2001) sintetizó las consecuencias de la repetición en más de cien estudios primarios realizados entre 1925 y 1999 y encontró más efectos negativos que positivos de esta estrategia de nivelación. Hattie (2009), como síntesis de 800 metaanálisis, llegó a la conclusión de que la repetición es una de las variables más importantes en los niveles de logro y cuantifica en un $16 \%$ el descenso que provoca en el rendimiento de los estudiantes, daño que solo es superado por el cambio de escuela y por tener más de una televisión en casa. Con estos resultados, es evidente la necesidad de un debate y de un replanteamiento de la cuestión que lleve a la identificación de nuevas estrategias que permitan reducir la repetición de curso y, por ende, sus efectos perversos, entre los que ocupa un lugar especial el riesgo de fracaso escolar.

La repetición es un fenómeno complejo y multicausal y existe una amplia literatura en los estudios PISA que muestran que tanto la repetición de curso (Carabaña, 2013; Cordero et al., 2014; Jheng, 2014; Pajares, 2005) como el riesgo de fracaso escolar (Calero, Escardibul \& Choi, 2012; Choi \& Calero, 2012; Cordero, Manchón \& Simancas, 2012; Márquez, 2016) están relacionados con diferentes variables contextuales. Estas se presentan en PISA 2015 agrupadas en torno a tres áreas de contenido: antecedentes del estudiante, procesos y medidas no cognitivas (figura 1).

Las variables de las áreas de procesos y productos no cognitivos se han estudiado más con relación al nivel de desempeño (Önder \& Uyar, 2018) que con relación a la repetición. Sin embargo, son abundantes los trabajos en los que han surgido variables del área de antecedentes del estudiante asociadas con nuestro objeto específico de estudio. Según la OCDE (2016b), incrementan la probabilidad de repetir: un menor nivel socioeconómico familiar, la nacionalidad (inmigrante) y el sexo (varón). Dentro del módulo 7, la categoría profesional de los padres (Calero et al., 2012), el tipo de familia monoparental (Fernández \& Rodríguez, 2008) y los recursos educativos del hogar (Cordero et al., 2014) son las variables que más afectan en la tasa de repetición. En cuanto a la etnia e inmigración (módulo 8), Pedraja, Santín y Simancas (2012) señalan que los inmigrantes de primera generación tienen más posibilidades de repetir. Además, las chicas tienen menor probabilidad de repetir (Méndez \& Cerezo, 2018) o de fracasar en el sistema educativo (Calero et al., 2012) y tienen una mayor posibilidad de alcanzar la excelencia en la competencia lingüística (Cordero et al., 2012). Sin embargo, esto no ocurre en ciencias (Rodríguez-Mantilla, Fernández-Díaz \& Jover, 2018), ni en matemáticas (Gamazo, Martínez-Abad, Olmos-Migueláñez \& RodríguezConde, 2018), disciplinas en las que rinden más los varones. También hay que tener presente la importancia del recorrido educativo en la primera infancia (módulo 9), ya que la escolaridad temprana se relaciona con un menor riesgo de fracaso escolar (Choi \& Calero, 2012). 
DELIA ARROYO RESINO, ISRAEL ALEXANDER CONSTANTE AMORES,

INMACULADA ASENSIO MUÑOZ

LA REPETICIÓN DE CURSO A DEBATE: UN ESTUDIO EMPÍRICO A PARTIR DE PISA 2015

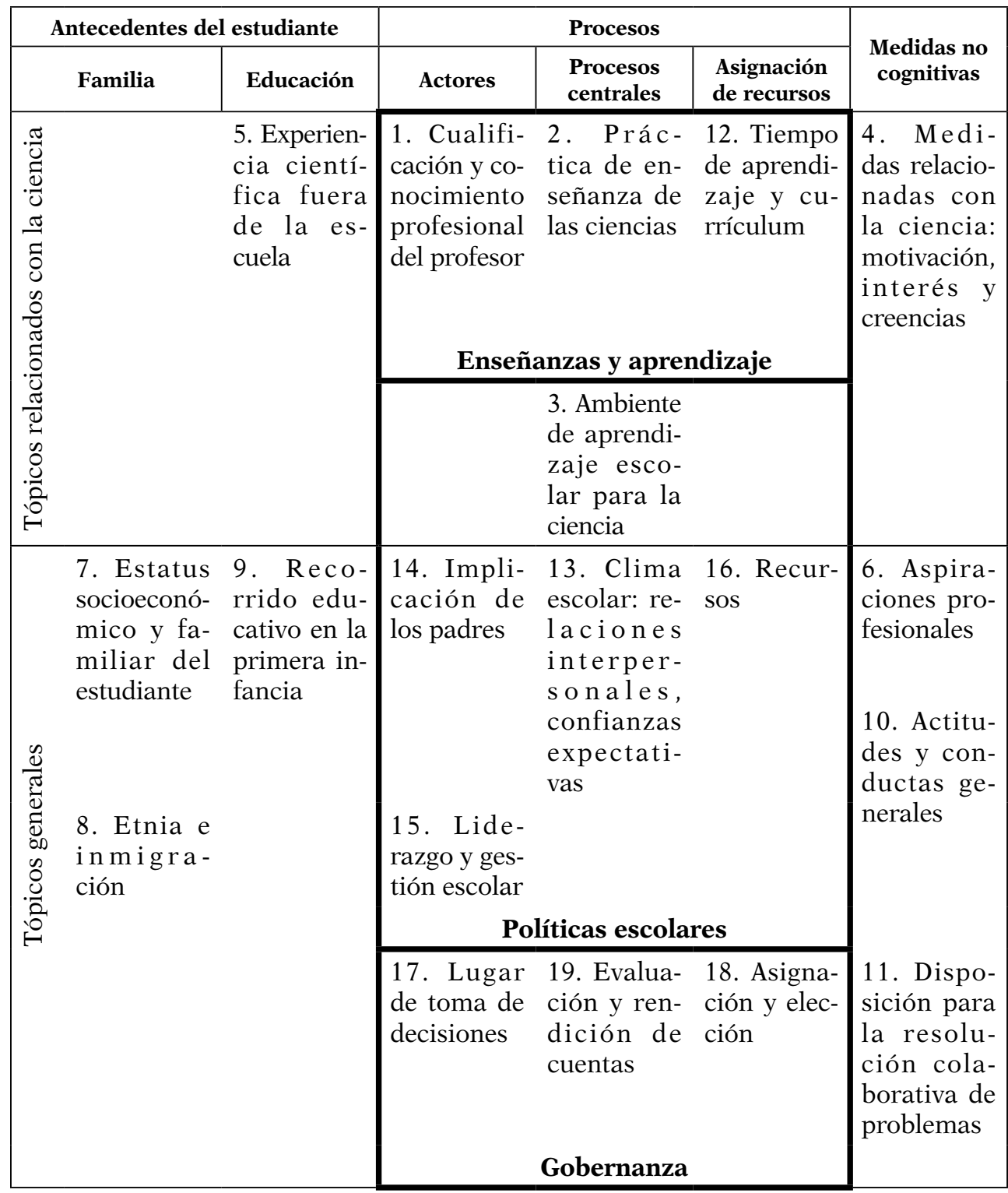

Figura 1. Estructura modular de las variables de contexto en PISA 2015

Fuente: OCDE 2016, p. 109

La literatura científica hasta el momento pone de manifiesto una serie de variables de contexto relacionadas con la repetición, pero estas normalmente no han sido incluidas en los modelos explicativos con un criterio objetivo, dado que no se ha jerarquizado su importancia mediante 
técnicas empíricas. Los resultados de investigación existentes entonces pueden depender en gran medida de que el grueso de la investigación se ha realizado desde las ciencias económicas. El objetivo general de este trabajo es ahondar en el estudio de las variables de contexto (antecedentes del estudiante, procesos y medidas no cognitivas) relacionadas con la repetición de curso para:

1. Identificar las variables de las diferentes áreas que mejor discriminan entre los perfiles del estudiante repetidor y no repetidor.

2. Ordenar dichas variables según su importancia relativa.

3. Seleccionar aquellas variables de contexto que más inciden en la probabilidad de repetir.

\section{MÉTODO}

Se trata de un estudio secundario con los resultados de PISA 2015 a través de una técnica de clasificación (para la identificación de perfiles y la selección de predictores) y un análisis de regresión logística, realizados mediante SPSS versión 23.

Se ha utilizado la base de datos referida al cuestionario de estudiantes españoles, clasificada según la variable objeto de estudio, de modo que se cuenta con 27.892 estudiantes no repetidores y 10.937 repetidores. Como variables predictoras se han seleccionado un total de 208 ítems, descartando aquellos que aportaban una información redundante (por ejemplo, repetir curso en primaria o secundaria, ya que se introduce el índice de repetición); asimismo se han eliminado predictores que manifiestan colinealidad con la variable dependiente, como es la edad. Además, se incluye el índice de inmigración, aunque en Choi et al. (2018) no resulta significativo, ya que según la literatura (Choi \& Calero, 2012; Cordero et al., 2014) es una variable relacionada con la repetición de curso.

Con la técnica de data mining de árboles de clasificación, que proporciona una serie de diagramas formados por nodos, que representan una segmentación de la población en subgrupos, de acuerdo con sus características en una serie de variables predictoras, que determinan su aportación en la variable criterio (Tourón, López-González, Lizasoain, García \& Navarro, 2018), se elaboran los perfiles de los dos grupos. Entre las alternativas existentes se ha seleccionado CART (Classification And Regression Trees), por ser un método de árbol binario numérico y exhaustivo (Román \& Levy, 2003), que da lugar a nodos de fácil interpretación (Castro \& Lizasoain, 2012) y que permite ordenar los predictores según su importancia normalizada. Se utiliza también como técnica de selección 
de las variables independientes, fijando como criterio de inclusión que su importancia normalizada fuera al menos de un 15\%. La técnica de clasificación se complementa en este estudio con un análisis de regresión logística con el fin de comparar ambas metodologías y conseguir un resultado más parsimonioso y robusto. Dado que el análisis de regresión es una técnica más exigente, antes de la especificación del modelo de regresión se comprobaron los supuestos de no colinealidad, de independencia de errores y la presencia de casos atípicos. Tras esta comprobación se redujo el número de variables seleccionadas. Los predictores con más de dos categorías fueron dicotomizados ( 0 y 1 ) para facilitar la interpretación. A partir del modelo de regresión también se calcularon los pronósticos, de acuerdo con la siguiente ecuación:

$$
\text { Logit (repetir = 1) }=B_{0}+B_{1}\left(X_{1}\right)+B_{2}\left(X_{2}\right)+B_{3}\left(X_{3}\right) \ldots B_{k}\left(X_{k}\right) \quad \text { Ecuación } 1
$$

En la ecuación $1, B_{0}$ se refiere a la constante del modelo y el resto de betas a los coeficientes asociados a cada una de las variables independientes donde, $X_{k}$ es el valor de su categoría correspondiente. Resolviendo la ecuación anterior, se obtiene el logit de repetir más alto y bajo según el valor de la categoría utilizada. Debido a la dificultad que supone la interpretación del logit, mediante la ecuación 2 se transformó dicha escala a probabilidades (Pardo \& Ruiz, 2013).

$$
\hat{\pi}=1 /\left(1+e^{-\log i t}\right)
$$

Ecuación 2

\section{RESULTADOS}

En primer lugar, con el algoritmo CART de árboles de decisión se obtienen las variables contextuales que mejor discriminan entre repetidores y no repetidores que se muestran en la figura 2. En la rama izquierda (nodos 1 y 4), se aprecia el perfil del alumnado repetidor. La mayor frecuencia de repetidores $(89,4 \%, \mathrm{n}=4426)$ se encuentra entre los estudiantes que se conforman con completar segundo ciclo de la ESO o menos (nodo 1) y que no han cursado ninguna asignatura de química el año anterior (nodo 4). Mientras que en la rama derecha (nodos 2, 5 y 9) se ofrece el perfil de los no repetidores. La frecuencia más alta de los no repetidores $(98,2 \%, \mathrm{n}=14040)$ se da entre los estudiantes que aspiran completar más del segundo ciclo de la ESO (nodo 2), que han asistido alguna asignatura de química el año anterior (nodo 5) y que no han asistido a Ciencias Aplicadas y Tecnología (CAT, de aquí en adelante) este año (nodo 9). 
INMACULADA ASENSIO MUÑOZ

LA REPETICIÓN DE CURSO A DEBATE: UN ESTUDIO EMPÍRICO A PARTIR DE PISA 2015

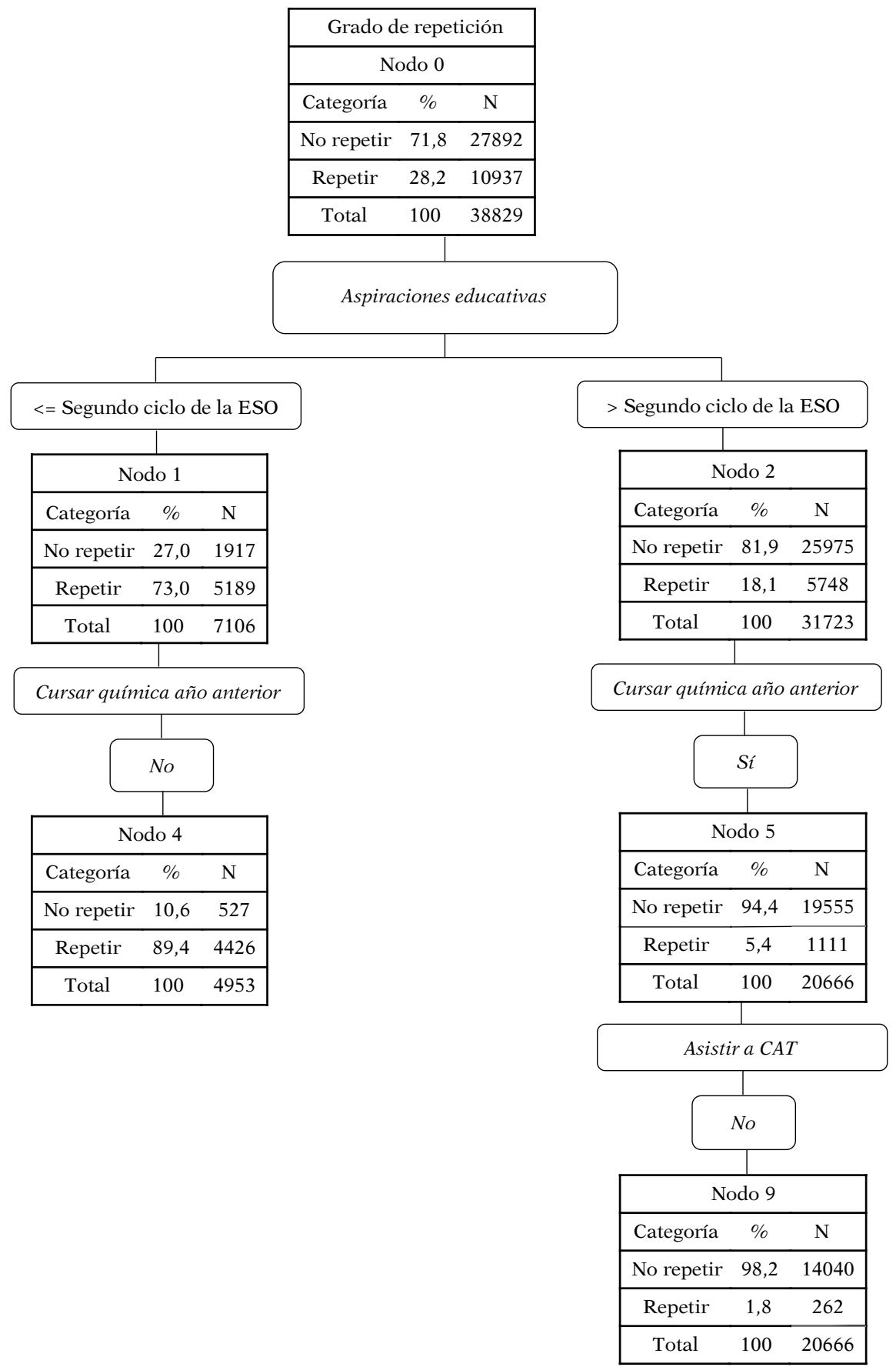

Figura 2. Árbol de decisión en el que se resumen los perfiles del estudiante repetidor y no repetidor 
En segundo lugar, con la misma técnica se procede a la selección de predictores. En la figura 3 se presentan las 14 variables independientes más importantes en la explicación de las diferencias entre repetidores y no repetidores, que cumplen con el criterio de inclusión de alcanzar una importancia normalizada superior a un $15 \%$. En la jerarquía obtenida con el algoritmo CART, el nivel de aspiraciones de los estudiantes alcanza el primer puesto, seguido por nueve variables curriculares, de modo que las variables de antecedentes del estudiante (estatus socioeconómico y familiar del estudiante y etnia e inmigración) aparecen en los últimos cuatro puestos del ranking (figura 3).

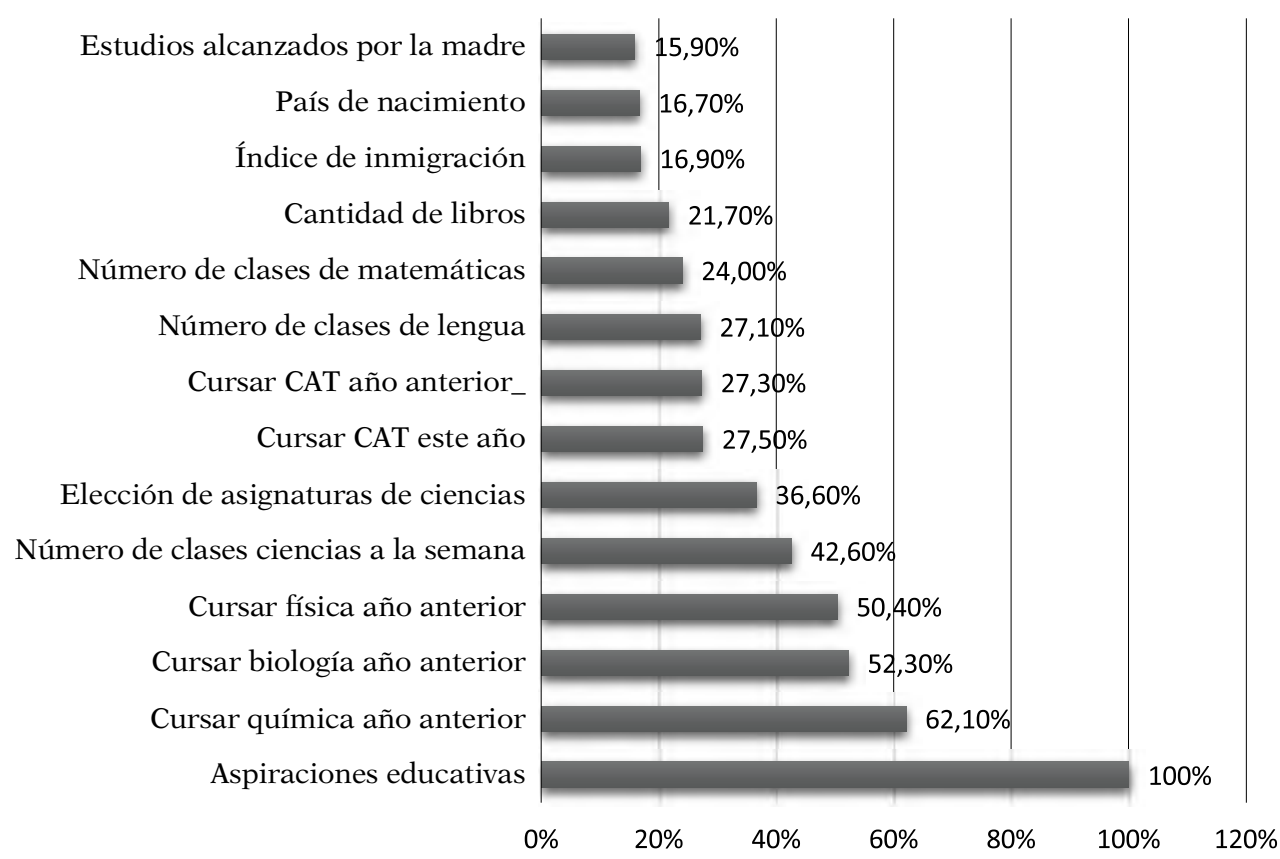

Figura 3. Importancia normalizada de las variables independientes respecto a la repetición

Una de las limitaciones de los árboles de clasificación es una cierta inestabilidad metodológica en la selección de predictores, provocada por el hecho de que en la partición recursiva influye no solo la decisión sobre qué variable se divide, sino también el punto de corte exacto utilizado en la división (López-Martín, Expósito-Casas, Carpintero \& Asensio, 2018; Strobl, Malley \& Tutz, 2009). Por esta razón, en el presente estudio se complementa el uso de esta herramienta exploratoria, con otra más confirmatoria como es el análisis de regresión. 
Antes de la regresión, se calculó la relación entre las variables independientes, se comprobó el supuesto de independencia de los errores, el de no colinealidad y se llevó a cabo un estudio de los casos atípicos. Los análisis de correlación entre los predictores mostraron una intensidad de relación inferior a 0,80, a excepción del coeficiente entre la variable cursar física año anterior y cursar química año anterior cuyo valor fue de 0,831. Siguiendo a Garbanzo (2014), que recomienda eliminar una de las variables cuando la correlación es superior a 0,70, se eliminó la variable cursar física año anterior, ya que era la que presentaba una menor importancia normalizada. De este modo, se cumplen los supuestos de independencia de los errores y de falta de multicolinealidad, dado que el valor Durbin-Watson obtenido se encuentra entre 1,5 y 2,5, los valores de tolerancia resultan superiores a 0,10 y los valores de FIV en ninguna de las variables superan el valor 10 (Pardo \& Ruiz, 2013). En cuanto al estudio de los casos atípicos no se eliminó ninguno, puesto que la distancia de Cook arroja valores inferiores a 1 (Field, 2009).

Tras la comprobación de los supuestos, se recodificaron algunas variables para facilitar la interpretación de los coeficientes, como se muestra en la tabla 1.

De todas las variables predictoras introducidas en el modelo de regresión, tan solo se mantuvieron aquellas cuyo coeficiente resultó significativo $(p<, 05)$. La tabla 2 ofrece una valoración del cambio que experimentó la desvianza del modelo nulo al incorporar las covariables finalmente seleccionadas.

La razón de verosimilitudes (chi-cuadrado) tiene un nivel menor que ,05 lo que nos permite concluir que todas las variables del modelo propuesto consiguen mejorar el ajuste del modelo nulo. En cuanto a la calidad del modelo (tabla 3), el estadístico $R^{2}$ de Nagelkerke indica que el modelo propuesto explica un $63,2 \%$ de la variabilidad de la variable repetición (44,4\% según Cox y Snell). 
Tabla 1

Valores de las variables del modelo de regresión inicial

\section{Variable criterio}

\begin{tabular}{|c|c|c|}
\hline & Variable & Valores \\
\hline \multicolumn{2}{|r|}{ Repetición de curso } & $\begin{array}{l}0=\text { No repite } \\
1=\text { Repite una o más veces en } \\
\text { primaria y/o secundaria** }\end{array}$ \\
\hline \multicolumn{3}{|c|}{ Variables predictoras } \\
\hline $\begin{array}{c}\text { Área de } \\
\text { contenidos }\end{array}$ & Variable & Valores \\
\hline $\begin{array}{l}\text { Medidas no } \\
\text { cognitivas }\end{array}$ & Aspiraciones educativas* & $\begin{array}{l}0 \text { = Menos o igual del segundo } \\
\text { ciclo de educación secundaria } \\
1 \text { = Más del segundo ciclo de } \\
\text { educación secundaria }\end{array}$ \\
\hline \multirow{8}{*}{ Proceso } & Cursar química año anterior* & $0=$ No cursó 1 = Sí cursó \\
\hline & Cursar biología año anterior* & 0 = No cursó 1 = Sí cursó \\
\hline & $\begin{array}{l}\text { Número de clases de ciencias } \\
\text { a la semana* }\end{array}$ & \\
\hline & $\begin{array}{l}\text { Elección de asignaturas de } \\
\text { ciencias* }\end{array}$ & 0 = No eligió 1 = Sí eligió \\
\hline & Cursar CAT este año* & $0=$ No cursó 1 = Sí cursó \\
\hline & Cursar CAT año anterior* & 0 = No cursó 1 = Sí cursó \\
\hline & Número de clases de lengua & \\
\hline & $\begin{array}{l}\text { Número de clases de mate- } \\
\text { máticas }\end{array}$ & \\
\hline \multirow{4}{*}{$\begin{array}{l}\text { Antecedentes } \\
\text { del } \\
\text { estudiante }\end{array}$} & Cantidad de libros* & $\begin{array}{l}0=\text { de } 0 \text { a } 25 \text { libros } * * * \\
1=\text { más de } 200 \text { libros }\end{array}$ \\
\hline & Índice de inmigración & $\begin{array}{l}0=\text { Nativo } \\
1 \text { = Primera y segunda genera- } \\
\text { ción }\end{array}$ \\
\hline & País de nacimiento & $0=$ España 1 = 0tro país \\
\hline & $\begin{array}{l}\text { Estudios alcanzados de la } \\
\text { madre* }\end{array}$ & $\begin{array}{l}0=\text { Estudios inferiores a ba- } \\
\text { chillerato } \\
1=\text { Bachillerato o más }\end{array}$ \\
\hline
\end{tabular}

$* p<, 05$.

** Se agrupan todos los repetidos en un solo grupo porque en las dos etapas educativas las causas de la repetición son semejantes (Carabaña, 2013).

*** Para maximizar varianza experimental, se toman solo los grupos extremos, por lo que se reduce la muestra. 
Tabla 2

Prueba ómnibus

\begin{tabular}{llccc}
\hline & & Chi-cuadrado & gl & Sig. \\
\hline \multirow{4}{*}{ Paso 1 } & Paso & 18243,756 & 9 &, 000 \\
& Bloque & 18243,756 & 9 &, 000 \\
& Modelo & 18243,756 & 9 &, 000 \\
\hline
\end{tabular}

Tabla 3

Resumen del modelo de regresión

\begin{tabular}{cccc}
\hline Paso & $\mathbf{- 2}$ log de la verosimilitud & $\boldsymbol{R}^{\mathbf{2}}$ de Cox y Snell & $\boldsymbol{R}^{\mathbf{2}}$ de Nagelkerke \\
\hline 1 & 19482,054 &, 444 &, 632 \\
\hline
\end{tabular}

En la tabla 4 se presentan los coeficientes del modelo de regresión logística. En ella se observa que cursar CAT este año es la única variable que se encuentra relacionada positivamente con la repetición.

Tabla 4

Estadísticos del modelo final de regresión

\begin{tabular}{lcccc}
\hline & B & E. T & Sig. & Exp(B) \\
\hline Aspiraciones educativas & $-2,028$ & 0,047 & 0,000 & 0,132 \\
Cursar química año anterior & $-1,632$ & 0,047 & 0,000 & 0,196 \\
Cursar biología año anterior & $-1,085$ & 0,045 & 0,000 & 0,338 \\
Número de clases de ciencias a la semana & $-0,127$ & 0,010 & 0,000 & 0,881 \\
Elección de asignaturas de ciencias & $-1,560$ & 0,037 & 0,000 & 0,210 \\
Cursar CAT este año & 1,504 & 0,040 & 0,000 & 4,500 \\
Cursar CAT el año anterior & $-0,236$ & 0,040 & 0,000 & 0,790 \\
Cantidad de libros & $-0,114$ & 0,012 & 0,000 & 0,892 \\
Estudios alcanzados por la madre & $-0,785$ & 0,038 & 0,000 & 0,456 \\
Constante & 3,204 & 0,073 & 0,000 & 24,621 \\
\hline
\end{tabular}

El valor exponencial de la constante indica que los estudiantes cuyas aspiraciones educativas sean completar segundo ciclo de educación secundaria o menos (aspiraciones $=0$ ), que no han cursado química (química $=0$ ) o biología (biología $=0$ ) el año anterior, que no han recibido clases de ciencias (clases ciencias $=0$ ), que no han elegido asignaturas relacionadas con ciencias 
$($ ciencias $=0)$, que no han recibido clases de CAT este año $(\mathrm{CAT}=1)$ ni el año anterior $(\mathrm{CAT}=0)$, que tienen como mucho 25 libros en casa (libros $=0$ ) y que son hijos de madres con estudios inferiores a bachillerato (estudios madre $=0$ ) tienen 24,621 veces más posibilidades de repetir.

En relación con las aspiraciones educativas se aprecia que su coeficiente tiene un signo negativo $(-2,028)$, por lo que la repetición es más probable en los estudiantes con menos aspiraciones. Concretamente, la odds de repetir entre los que esperan completar más del segundo ciclo de educación secundaria (aspiraciones $=1$ ) es un 86,8\% menor que los que esperan completar segundo o menos (aspiraciones $=0$ ). Respecto a cursar química el año anterior el valor 0,196, indica que la posibilidad de repetir entre los que han asistido (química $=1$ ) es un 80,4\% menor que entre los que no han asistido (química $=0$ ). Cursar biología el año anterior disminuye la probabilidad de repetir, ya que el coeficiente 0,338 muestra que la odds de repetir en los que han cursado la asignatura (biología $=1$ ) es un $66,2 \%$ menor que entre los que no (biología $=0$ ). El signo negativo de la variable número de clases de ciencias a la semana muestra que la probabilidad de repetir disminuye cuando aumenta el número de clases, de modo que, por cada clase de más que reciban, la odds de repetir disminuye un 11,9\%. La elección de asignaturas de ciencias disminuye la probabilidad de repetir, pues la odds de repetir en los que han elegido alguna asignatura de ciencias $($ ciencias $=1$ ) es un $79 \%$ menor que entre los que no la han elegido (ciencias $=0$ ). Cursar CAT este año $(\mathrm{CAT}=1)$ incrementa la probabilidad de repetir 4,5 veces. Por el contrario, cursar CAT el año anterior disminuye la probabilidad de repetir, en un $21 \%$, respecto a los que no han cursado ciencias y tecnología (CAT $=0$ ). Así mismo, en cuanto a la variable cantidad de libros los resultados muestran que la probabilidad de repetir disminuye cuando se tienen más de 200 libros en casa. Concretamente, para los estudiantes con más de 200 libros en el hogar (libros $=1$ ) la odds de repetir es un 10,8\% menos que los que tienen como mucho 25 (libro $=0$ ). Por último, el valor 0,456 indica que la odds de repetir en los estudiantes cuya madre tiene al menos bachillerato (estudios madre $=1$ ) es un $54,4 \%$ menor que entre los estudiantes cuya madre tiene estudios inferiores a ese nivel (estudios madre $=0$ ).

En cuanto a los pronósticos del modelo, la probabilidad más baja de repetir se da entre los estudiantes cuyas aspiraciones educativas sean completar más del segundo ciclo de educación secundaria (aspiraciones $=1$ ), que han cursado el año anterior química (química $=1$ ) o biología (biología $=1$ ), que más clases de ciencias reciben a la semana (clases ciencias $=10$ ), que han elegido las asignaturas de ciencias (ciencias $=1$ ), que no realizan clases de CAT este año $(\mathrm{CAT}=0)$ pero sí el año anterior (CAT $=1)$, que poseen más de 200 libros en el hogar (libros $=1)$ y cuyas madres han cursado al menos bachillerato (estudios madre $=1$ ). En estos estudiantes la probabilidad de repetir es muy baja $(0,004)$. 
Por el contrario, para los que tienen la aspiración educativa de completar segundo ciclo de educación secundaria o menos (aspiraciones $=0$ ), que no han cursado química (química $=0$ ) o biología $($ biología $=0$ ) el año anterior, que no reciben clases de ciencias (clases ciencias $=0$ ), que no han elegido asignaturas de ciencias (ciencias $=0$ ), que realizan clases de CAT este año $($ CAT $=1)$, pero no el año anterior $(\mathrm{CAT}=0)$, que tienen menos o igual a 25 libros en el hogar (libros $=0$ ) y que los estudios alcanzados de la madre son inferiores a bachillerato (estudios madre $=0$ ), la probabilidad de repetir es del 0,991.

En cuanto al valor predictivo, los modelos resultantes de la técnica de clasificación y de la regresión logística son muy similares como se puede observar en las tablas 5 y 6 , siendo el porcentaje de coincidencia entre los valores observados y pronosticados por los modelos muy altos en el grupo de no repetidores y algo menos favorable en el de repetidores. El comportamiento del modelo CART es algo mejor en este último grupo, aunque la técnica de regresión es más parsimoniosa, al incluir solo 9 de las 14 variables que emergieron con CART.

Tabla 5

Matriz de confusión (árboles de decisión)

\begin{tabular}{lccc}
\hline \multirow{2}{*}{ Observado } & \multicolumn{3}{c}{ Pronosticado } \\
\cline { 2 - 4 } & $\begin{array}{c}\mathbf{0} \\
\text { No repetidor }\end{array}$ & $\begin{array}{c}\mathbf{1} \\
\text { Repetidor }\end{array}$ & Porcentaje correcto \\
\hline 0 No repetidor & 25.558 & 2.334 & $91,6 \%$ \\
1 Repetidor & 2.371 & 8.566 & $78,3 \%$ \\
\hline Porcentaje global & & & $87,9 \%$ \\
\hline
\end{tabular}

Tabla 6

Método de crecimiento: CART

Variable dependiente: repetición. Matriz de confusión (regresión logística)

\begin{tabular}{|c|c|c|c|c|c|}
\hline & \multirow{3}{*}{ Observado } & & \multicolumn{3}{|c|}{ Pronóstico } \\
\hline & & & \multicolumn{2}{|c|}{ Repetición } & \multirow[b]{2}{*}{$\%$ correcto } \\
\hline & & & $\begin{array}{c}0 \\
\text { No repetir }\end{array}$ & $\begin{array}{c}1 \\
\text { Repetir }\end{array}$ & \\
\hline \multirow{3}{*}{ Paso 1. ${ }^{\circ}$} & \multirow{2}{*}{ Repetición } & 0 No repetir & 20.222 & 1.611 & 92,6 \\
\hline & & 1 Repetir & 2.478 & 6.722 & 73,1 \\
\hline & Porcentaje global & & & & 86,8 \\
\hline
\end{tabular}

a. El valor de corte es 0,500

Nota. Se produce una disminución de muestra porque al dicotomizar la variable cantidad de libros se han seleccionado solo los grupos extremos (ver tabla 1) 


\section{DISCUSIÓN Y CONCLUSIONES}

Con este trabajo se pretendía profundizar en el estudio de las variables de contexto (antecedentes del estudiante, procesos y medidas no cognitivas) en PISA 2015, que se encuentran relacionadas con la repetición de curso, con la finalidad última de valorar su importancia y así obtener evidencias en las que basar las decisiones educativas para reducir la incidencia de este fenómeno en nuestro sistema educativo.

Respecto al primer objetivo, consistente en identificar las variables que mejor discriminan entre el perfil del estudiante repetidor y del no repetidor, se puede concluir que los ítems del cuestionario de contexto de PISA referidos a aspiraciones educativas y cursar química el año anterior son los predictores que mejor definen y diferencian a dichos estudiantes. Estas variables aparecen también como las más importantes en el ranking que aporta CART. Dichos resultados se encuentran en sintonía con los obtenidos por Santos, Godás y Lorenzo (2012), que destacan la importancia de las aspiraciones educativas en el estudiante repetidor, con Simons-Morton y Chen (2009), que relacionan el fracaso escolar con las aspiraciones educativas del estudiante o con López-Martín et al. (2018) que señalan la repetición como la principal variable relacionada con el desempeño en ciencias y las aspiraciones como una de las que definen el perfil de los estudiantes de alto rendimiento.

Con relación al segundo objetivo específico de jerarquizar las variables predictoras por orden de importancia, queda claro, que, incluyendo en el modelo simultáneamente diferentes tipos de variables de diversos módulos, las de tipo socioeconómico y familiar ocupan puestos de menor valor predictivo, aunque en la literatura son las que aparecen con mayor frecuencia (Carabaña, 2013; Choi et al., 2018; Cordero et al., 2014; Simons et al., 2009).

En cuanto al objetivo de seleccionar los predictores más relevantes, complementando los resultados de CART con los aportados por el análisis de regresión logística, se observa que la probabilidad de repetir es menor cuando los estudiantes tienen aspiraciones educativas más altas (superior a segundo ciclo de educación secundaria), cuando asisten a clases de química o biología el año anterior, cuantas más horas de ciencias realicen a la semana, cuando los estudiantes eligen asignaturas de ciencias y cuando no cursan la asignatura de CAT este año. Sin embargo, si la realizaron el año pasado, su probabilidad de repetir es mayor que la de los estudiantes que no la cursaron. También el número de libros en el hogar es importante, ya que, quienes poseen menos tienen una mayor probabilidad de repetir. En esta línea, Cordero, et al. (2014) y Evans, Kelley, Sikora y Treiman, (2010) 
obtienen evidencia del efecto negativo de contar con recursos culturales escasos. Por último, los estudios alcanzados por la madre resultan relevantes en nuestra investigación, puesto que el alumnado tiene mayor probabilidad de repetir si sus madres solo cuentan con estudios inferiores a bachillerato. Sin embargo, no ocurre lo mismo con el nivel educativo del padre que sí resulta importante en otros trabajos (Carabaña, 2013; Pérez-Diaz, Rodríguez y Fernández, 2008). Respecto a las variables de procesos, apenas se han encontrado investigaciones que demuestren la relación que existe entre ellas y la repetición y/o el riesgo de fracaso escolar, aunque Birbili (2005) y Calero (2006) ponen de manifiesto el impacto que el currículum y la organización escolar tienen en la eficacia del sistema educativo.

A la vista de estos resultados y siguiendo la clasificación realizada por PISA 2015 sobre las variables de contexto, la mayoría de los predictores que han surgido de la metodología empleada en este trabajo se encuentran relacionados con el área de procesos, a pesar de que se ha encontrado una extensa literatura (Calero et al., 2012; Choi, 2017; Choi et al., 2018; Cordero et al., 2012; Pedraja et al., 2012) que indique la importancia que tiene el área de antecedentes del estudiante en la repetición o en el riesgo de fracaso escolar. En el presente estudio se obtiene que dichas variables son relevantes, ya que, de los nueve predictores finalmente obtenidos, dos (estudios alcanzados por la madre y cantidad de libros) se encuentran relacionados con esta área. Sin embargo, no son los más importantes. Nuestros resultados destacan la importancia de las aspiraciones educativas, pero sobre todo resultan mayoritarias las variables vinculadas con el tiempo de aprendizaje y currículum en ciencias. Por consiguiente, este hallazgo complementa y matiza muchos de los resultados obtenidos en las investigaciones de los autores mencionados anteriormente y los contradice en el sentido de que se basan indirectamente en la teoría del capital cultural de Bourdieu (1986), que defiende la preponderancia que tiene el origen social (estudios y ocupación de los padres, recursos educativos del hogar, etc.) en el rendimiento académico. PISA, como iniciativa de una organización con fines fundamentalmente económicos, es foco de interés principal para sociólogos y economistas de la educación, pero los resultados de esta evaluación internacional también dan pistas a los investigadores educativos de que las variables de procesos son relevantes. La preponderancia de las variables socioeconómicas, si se incluyen variables de diferente tipo en los modelos estadísticos, no está tan clara cuando se trata de explicar la repetición.

La importancia de un producto no cognitivo, como es el nivel de aspiraciones, y de variables del área de procesos, debe tenerse en cuenta tanto por parte de la escuela, como de las políticas educativas. En este sentido, parece coherente trabajar en el aula el nivel de aspiraciones, así 
como desarrollar actividades que refuercen el interés y los conocimientos en ciencias motivando a los estudiantes en dicha área, de tal manera que sean ellos mismos los que elijan cursar estas asignaturas. Dada la importancia que las expectativas académicas del estudiante tienen en la repetición, sería adecuado que desde el centro educativo se trabajara en la mejora del autoconcepto académico del estudiante, tal y como indican Ibarra-Aguirre (2014), Ibarra y Jacobo (2016) y Serrano, Mérida y Tabernero (2016). Además, por la importancia que ha alcanzado el número de libros, se avala la conveniencia de políticas que los faciliten a las familias y de actividades pedagógicas de fomento de la lectura.

\section{APORTACIONES Y LIMITACIONES}

La principal aportación del estudio, en línea con lo obtenido por Asensio et al. (2018) y López-Martín et al. (2018), consiste en que, junto con las aspiraciones educativas del estudiante (producto no cognitivo), las variables más importantes son del área de procesos de enseñanzaaprendizaje en ciencias, que es el foco de atención en la edición de PISA 2015. Esto se desvincula de los resultados de los trabajos realizados por sociólogos o economistas (Carabaña, 2013; Choi et al., 2018; Cordero et al., 2014; Simons et al., 2009), en los que la repetición se asocia más con variables de antecedentes del estudiante de tipo personal y familiar.

Este trabajo también representa una aportación desde el punto de vista metodológico, por tres razones. Primero, porque la utilización de los árboles de decisión, como técnica de selección previa de los predictores a incluir en los modelos de regresión, resulta novedosa y altamente útil para reducir errores de especificación (Asensio et al., 2018). Segundo, porque las técnicas arborescentes son muy versátiles y especialmente adecuadas cuando se trabaja con una gran cantidad de predictores, permitiendo el uso simultáneo de variables medidas en diferentes escalas. La versatilidad es una cualidad esencial cuando se trabaja con los cuestionarios de contexto de PISA que son cada vez más complejos (González-Such, Sancho-Álvarez \& Sánchez-Delgado, 2016). Tercero, porque este estudio aporta evidencia de que los árboles de clasificación y el modelo de regresión logística son técnicas complementarias y robustas.

Como prospectiva de investigación, aunque el cuestionario del estudiante es el más informativo del desempeño en ciencias (Asensio et al., 2018), sería interesante estudiar la importancia de los ítems de los otros cinco cuestionarios de contexto de PISA (trayectoria académica, uso de las TIC, cuestionario de la familia, del centro y del profesor) en relación con la repetición. 
Por último, como limitación hay que advertir que los resultados obtenidos no permiten concluir en términos de causalidad. Para ello, sería necesario realizar modelos de ecuaciones estructurales o de estructuras de covarianza, idóneos para analizar el tipo (directa, indirecta o no causal) y el sentido de las relaciones en diseños no experimentales. 


\section{REFERENCIAS BIBLIOGRÁFICAS}

Asensio, I., Carpintero, E., López, E., y Expósito, E. (2018). ¿Cuánto oro hay entre la arena? Minería de datos con los resultados de España en PISA 2015. Revista Española de Pedagogía, 270, 225-245. doi: https://doi.org/10.22550/ REP76-2-2018-02.

Benito, A. (2007). La LOE ante el fracaso, la repetición y el abandono escolar. Revista Iberoamericana de Educación, 43, 1-11.

Birbili, M. (2005). Constants and contexts in pupil experience of schooling in England, France and Denmark. European Educational Research Journal, 4(3), 313-320. doi:10.1080/03050060126690.

Bourdieu, P. (1986). The Forms of Capital. In J.G. Richardson (Ed.), Handbook of theory and research for the sociology of education (pp. 241-258). Nueva York, USA: Greenwood Press.

Calero, J. (2006). Desigualdades tras la educación obligatoria: nuevas evidencias. Documento de Trabajo de la Fundación Alternativas, 83(6), 56-78.

Calero, J., Escardibul, J.O., y Choi, Á. (2012). El fracaso escolar en la Europa mediterránea a través de PISA-2009: radiografía de una realidad latente. Revista Española de Educación Comparada, 19, 69-104. doi: https:// doi.org/10.5944/reec.19.2012.7578.

Carabaña, J. (2013). Repetición de curso y puntuaciones PISA ¿Cuál causa cuál? En Instituto Nacional de Evaluación Educativa (Ed.), PISA 2012: Programa para la evaluación de los alumnos, informe español (volumen II: análisis secundario). Madrid: INEE.

Castro, M. y Lizasoain, L. (2012). Las técnicas de modelización estadística en la investigación educativa: minería de datos, modelos de ecuaciones estructurales y modelos jerárquicos lineales. Revista Española de Pedagogía, 251, 131-148.

Choi, Á. (2017). Efectos de la repetición de curso y alternativas. Recuperado de https://bit.ly/2J9egRJ.

Choi, Á., y Calero, J. (2012). Determinantes del riesgo de fracaso escolar en España en PISA-2009 y propuestas de reforma. Revista de Educación, 362, 562-593. doi: 10.4438/1988-592X-RE-2013-362-242.

Choi, Á., Gil, M., Mediavilla, M. \& Valbuena, J. (2018). Predictors and Effects of Grade Repetition. Revista de Economía Mundial, 48, 21-42.

Cordero, J. M., Crespo, E., y Pedraja, F. (2013). Rendimiento educativo y determinantes según PISA: Una revisión de la literatura en España. Revista de Educación, 362. Recuperado de https://bit.ly/2N64wdp.

Cordero, J.M., Manchón, C., y Simancas, R. (2012). Análisis de los condicionantes del rendimiento educativo de los alumnos españoles en PISA 2009 mediante técnicas multinivel. Presupuesto y Gasto Público, 67, 71-96.

Cordero, J., Machón, C., y Simancas, R. (2014). La repetición de curso y sus factores condicionantes en España. Revista de Educación, 365, 12-37. doi: 10.4438/1988-592X-RE-2014-365-263.

Crespo, E. (2018). Fracaso escolar español: Una comparativa internacional con datos de PISA, En Catálogo de investigación joven en Extremadura (volumen II, pp. 84-89). Servicio de Publicaciones. 
European Commission (2010). Europe 2020 A European Strategy For Smart, Sustainable and Inclusive Growth. Brussels, Bélgium: European Commission.

Evans, M. D., Kelley, J., Sikora, J., \& Treiman, D. J. (2010). Family scholarly culture and educational success: Books and schooling in 27 nations. Research in social stratification and mobility, 28(2), 171-197.

Fernández, J.J. y Rodríguez, J. C. (2008). Los orígenes del fracaso escolar en España: un estudio empírico. Mediterráneo Económico, $14,323-349$.

Ferrão, M.E., Costa, P.M., \& Matos, D.A.S. (2017). The relevance of the school socioeconomic composition and school proportion of repeaters on grade repetition in Brazil: a multilevel logistic model of PISA 2012. Largescale Assessments in Education, 5(1), 5-7. doi. 10.1186/s40536-017-0036-8.

Field, A. (2009). Discovering Statistics Using SPSS (Introducing Statistical Methods). London, UK: Sage Publications.

Gamazo, A., Martínez-Abad, F., OlmosMigueláñez, S., y Rodríguez-Conde, M. J. (2018). Evaluación de factores relacionados con la eficacia escolar en PISA 2015. Un análisis multinivel. Revista de Educación, 379, 56-84. doi: 10.4438/1988-592X-RE-2017-379-369.

Garbanzo, G.M. (2014). Factores asociados al rendimiento académico tomando en cuenta el nivel socioeconómico: Estudio de regresión múltiple en estudiantes universitarios. Revista Electrónica Educare, 18(1), 119-154.

González-Such, J., Sancho-Álvarez, C., y Sánchez-Delgado, P. (2016).
Cuestionarios de contexto PISA: un estudio sobre los indicadores de evaluación. RELIEVE, 22(1), M7. doi:http://dx.doi.org/10.7203/ relieve.22.1.8429

Hattie, J. (2009). Visible Learning: A Synthesis of Over 800 Meta-Analyses Relating to Achievement. Nueva York, USA: Taylor \& Francis.

Ibarra, E. y Jacobo, H.M. (2016). La evolución del autoconcepto académico en adolescentes. Revista mexicana de investigación educativa, 21(68), 45-70.

Ibarra-Aguirre, E. (2014). El autoconcepto en la adolescencia. Un estudio evolutivo. [Tesis doctoral]. Universidad Autónoma de Sinaloa, México.

Jacob, B.A., \& Lefgren, L. (2009). The Effect of Grade Retention on High School Completion. American Economic Journal: Applied Economics, 1(3), 33-58.

Jheng, Y. (2014). Does grade repetition work? Who repeats grades? Evidence from the scores of reading literacy of PISA 2009 of Macau. Jiaoyu Yanjiu Yuekan. Journal of Education Research, 242, 97-114.

Jimerson, S. R. (2001). Meta-analysis of grade retention research: Implications for practice in the 21 st century. School Psychology Review, 30, 420-437.

Ley Orgánica 1/1990, de 3 de octubre, Ordenación General del Sistema Educativo. BOE núm. 238 (4 de octubre de 1990), 28927-28942.

Ley Orgánica 10/2002, de 23 de diciembre, Calidad de la Educación. BOE núm. 307 (24 de diciembre de 2002), 45188-45220.

Ley Orgánica 8/2013, de 9 de diciembre, para la Mejora de la Calidad Educativa. BOE. 
López-Martín, E., Expósito-Casas, E., Carpintero, E., y Asensio, I. (2018). ¿Qué nos dice PISA sobre la enseñanza y el aprendizaje de las Ciencias? Una aproximación a través de árboles de decisión. Revista de Educación, 382, 133-161. doi: 10.4438/1988-592X-RE2018-382-395

Manacorda, M. (2012). The Cost of Grade Retention. The Review of Economics and Statistics, 94(2), 596-606.

Márquez, C. (2016). Factores Asociados al Fracaso Escolar en la Educación Secundaria de Huelva. Revista Iberoamericana sobre Calidad, Eficacia y Cambio en Educación, 14(3), 131144. doi:10.15366/reice2016.14.3.007.

Méndez, I. y Cerezo, F. (2018). La repetición escolar en educación secundaria y factores de riesgo asociados. Educación XX1, 21(1), 4162. doi: 10.5944/educXX1.13717.

Önder, E., \& Uyar, S. (2018). Factors affecting the academic achievement in socioeconomically disadvantaged students. Pegem Egitim Ve Ogretim Dergisi, 8(2), 253-280.

OCDE Organización para la Cooperación y el Desarrollo Económicos (2012). PISA 2012. Programa para para la evaluación internacional de los alumnos. Informe español. Madrid: Instituto Nacional de Evaluación Educativa. Subdirección General de Documentación y Publicaciones.

OCDE Organización para la Cooperación y el Desarrollo Económicos (2016a). PISA 2015. Programa para la evaluación internacional de los alumnos. Informe español. Madrid: Instituto Nacional de Evaluación Educativa. Subdirección General de Documentación y Publicaciones.

OCDE Organización para la Cooperación y el Desarrollo Económicos (2016b).
PISA 2015. Resultados clave. París, France: OECD Publishing.

Pajares, R. (2005). Resultados en España del estudio PISA 2000: conocimientos $y$ destrezas de los alumnos de 15 años. Madrid: INECSE.

Pardo, M. y Ruiz, M. (2013). Análisis de Datos en Ciencias Sociales y de la Salud III. Madrid: Síntesis.

Pedraja, F., Santín, D., y Simancas, R. (enero, 2012). El impacto de la concentración de inmigrantes sobre la repetición de curso en España. XX Encuentro de Economía Pública, Sevilla.

Pérez-Díaz, V.P., Rodríguez, J.C., y Fernández, J.J. (2009). Educación y familia: los padres ante la educación general de sus hijos en España. Recuperado de https://bit.ly/2veEJs3.

Rodríguez-Mantilla, J.M., FernándezDíaz, M.J., y Jover, G. (2018). PISA 2015: Predictores del rendimiento en Ciencias en España. Revista de Educación, 380, 75-102. doi: 10.4438/1988-592X-RE-2017-380-373.

Román, M.V. y Lévy, J.P. (2003). Clasificación y segmentación jerárquica. En J.-P. Lévy y J. Valera (Dietrs), Análisis Multivariable para las Ciencias Sociales (pp. 567-630). Madrid: Pearson Prentice Hall.

Santos, M., Godás, A., y Lorenzo, M. (2012). El perfil del alumnado repetidor y no repetidor en una muestra de estudiantes españoles y latinoamericanos: Un estudio sobre los determinantes de sus logros académicos. Estudios Sobre Educación, 23, 43-62.

Serrano, A., Mérida, R. \& Tabernero, M. (2016). La autoestima infantil, la edad, el sexo y el nivel socioeconómico como predictores del rendimiento 
académico. Revista de investigación en educación, 14 (1), 53-66.

Simons-Morton, B. \& Chen, R. (2009). Peer and parent Influences on school engagement among early adolescents. Youth \& Society, 41(1), 3-25. doi: 10.1177/0044118X09334861.

Strobl, C., Malley, J., \& Tutz, G. (2009). An introduction to recursive partitioning: Rationale, application, and characteristics of classification and regression trees, bagging, and random forest. Psychological Methods, 14(4). 323-348. doi: 10.1037/a0016973

Tourón, J., López-González, E., Lizasoain, L., Navarro, E., y García, M. J. (2018). Alumnado español de alto y bajo rendimiento en ciencias en PISA 2015: análisis del impacto de algunas variables de contexto. Revista de Educación, 380, 156-184. doi: 10.4438/1988-592X-RE-2017-380-376 


\section{PERFIL ACADÉMICO Y PROFESIONAL DE LOS AUTORES}

Delia Arroyo Resino. Licenciada de Pedagogía (UCM) con premio extraordinario y Máster en Estudios Avanzados de Pedagogía (UCM) y Metodología de las Ciencias del Comportamiento y la Salud (UCM, UAM y UNED) y Doctorado Internacional en Educación (UCM). Miembro del Grupo de Investigación Medida y Evaluación de Sistemas Educativos (Grupo MESE) desde el año 2014. Actualmente docente de la Universidad Internacional de la Rioja (UNIR) del departamento Métodos de Investigación y Diagnóstico en Educación (MIDE).

Israel Alexander Constante Amores. Graduado en Pedagogía y Máster de Investigación en Educación en la Universidad Complutense de Madrid. Actualmente cursa el Programa de Doctorado en Educación en el Departamento de Investigación y Psicología en Educación de la UCM. Su principal línea de interés es el estudio del rendimiento académico del alumnado.

Inmaculada Asensio Muñoz. Doctora en Pedagogía (UCM) con premio extraordinario. Es profesora titular del Departamento de Investigación y Psicología en Educación e imparte docencia en la Facultad de Educación. Como miembro del Grupo de Investigación Medida y Evaluación de Sistemas Educativos (Grupo MESE), su línea de trabajo principal son los métodos de investigación en Educación.

Dirección de los autores: Delia Arroyo Resino

C/ Luis de Hoyos Sainz, 146, 5c

28030 Madrid

E-mail: delia.arroyo@unir.net

Israel Alexander Constante Amores

Inmaculada Asensio Muñoz

Universidad Complutense de Madrid

Facultad de Formación del profesorado

C/Rector Royo Villanova, s/n

28040 Madrid

E-mail: iconstan@ucm.es

macu@edu.ucm.es

Fecha Recepción del Artículo: 01. Septiembre. 2018

Fecha Modificación del Artículo: 25. Noviembre. 2018

Fecha Aceptación del Artículo: 30. Noviembre 2018

Fecha Revisión para Publicación: 01. Febrero. 2019 